\title{
En busca del placer... Una perspectiva de género
}

À la recherche du plaisir... Une perspective de genre

Searching for pleasure... A gender perspective

\section{Céline Geffroy}

\section{OpenEdition}

Journals

Edición electrónica

URL: http://journals.openedition.org/bifea/8051

DOI: $10.4000 /$ bifea.8051

ISSN: 2076-5827

Editor

Institut Français d'Études Andines

Edición impresa

Fecha de publicación: 1 diciembre 2016

Paginación: 373-388

ISSN: 0303-7495

Referencia electrónica

Céline Geffroy, «En busca del placer... Una perspectiva de género », Bulletin de l'Institut français d'études andines [En línea], 45 (3) | 2016, Publicado el 08 diciembre 2016, consultado el 05 noviembre 2020. URL : http://journals.openedition.org/bifea/8051 ; DOI : https://doi.org/10.4000/bifea.8051

\section{(c) (i) $\odot$}

Les contenus du Bulletin de l'Institut français d'études andines sont mis à disposition selon les termes de la licence Creative Commons Attribution - Pas d'Utilisation Commerciale - Pas de Modification 4.0 International. 


\title{
En busca del placer... Una perspectiva de género
}

\author{
Céline Geffroy*
}

\begin{abstract}
Resumen
El objetivo de este artículo es indagar sobre las condiciones de acceso al placer sexual, focalizando su análisis en la complejidad de las relaciones de género y generacionales, donde siguen prevaleciendo las relaciones de dominación, a partir de los resultados obtenidos de un trabajo de campo etnográfico en zonas rurales y urbanas de Cochabamba (Bolivia). Se hará un enfoque en la asociación entre las violencias domésticas y la sexualidad, enmarcada en este contexto patriarcal estatal y gubernamental. Finalmente, se reflexionará sobre cómo las mujeres encuentran caminos para expresar sus deseos y buscar formas de placer.
\end{abstract}

Palabras clave: placer sexual, sexualidad, género, dominación masculina, violencia doméstica

\section{À la recherche du plaisir... Une perspective de genre}

\section{Résumé}

À partir des résultats obtenus lors d'un travail de terrain en ethnographie dans des zones rurales de Cochabamba (Bolivie), cet article traite des conditions d'accès au plaisir sexuel, en mettant l'accent sur les complexités des relations de genre et générationnelles, où continuent de prévaloir les relations de domination. On insistera sur l'association entre les violences domestiques et la sexualité, prise dans un contexte patriarcal d'État et de gouvernement. Finalement, on portera l'analyse sur les moyens imaginés par les femmes pour exprimer leurs désirs et chercher d'autres formes de plaisir.

Mots-clés : plaisir sexuel, sexualité, genre, domination masculine, violence domestique 


\section{Searching for pleasure... A gender perspective}

\section{Abstract} Based on an ethnography in rural and urban areas of Cochabamba, the goal of this article is to and generational relations where the relations of domination prevail. The article emphasizes ofsociation between domestic violence and sexuality, framed within this governmental context and patriarchal state. Finally, the analysis will focus on the biases experienced by women when expressing their desires and their search for other forms of pleasure.

Keywords: sexual pleasure, sexuality, gender, male dominance, domestic violence

El espasmo sexual es la sensación que procura más placer al ser humano —afirma Tiger (2003) en su libro sobre la búsqueda de los placeres-. Para Balandier (1984), la sexualidad es un hecho social total, ya que los actos sexuales abarcan una dimensión a la vez emocional, social, política, legal, étnica, generacional, fisiológica y hasta religiosa. Sin embargo, rodeados de misterios, de normas y de prohibiciones, la sexualidad y el placer son siempre temas difíciles de tratar, a diferencia de otras necesidades vitales como comer, beber o dormir... Aquí reside el interés por un estudio antropológico que aclare un aspecto tan esencial de la vida cotidiana poco estudiado, particularmente en el ámbito boliviano andino.

La elección de Bolivia no es neutra puesto que, como numerosos países emergentes, conoce, desde hace varios años, profundas transformaciones políticas, económicas y sociales, observables en tiempos muy breves. La noción misma de placer no escapa de estas mutaciones. Estos cambios están particularmente ligados al acceso masivo a la economía de mercado, a las migraciones internas, pero sobre todo internacionales, a la urbanización galopante (ella misma fruto de un modo de vida cambiante) y también a la influencia de las telenovelas. Entonces, dentro de este nuevo panorama, el acceso al placer está cambiando, particularmente entre las nuevas generaciones, sobre todo citadinas, que parecen vivir su sexualidad privilegiando el placer de ambos en la pareja.

A pesar de estos cambios, se plantea la hipótesis de que las discriminaciones sexistas y patriarcales hacia las mujeres, así como las restricciones y los tabúes, siguen afectando la manifestación y la percepción del placer, o por lo menos su exteriorización; la búsqueda de placer sexual no siempre tiene lugar dentro de un contexto de equilibrio entre los sexos. Según Joël Candau (2017), ciertas matrices culturales pueden inducir ciertas formas de felicidad mientras que otras no serían propicias para favorecerlas. Apoyándose en esta idea, se puede suponer que, en Bolivia, las relaciones machistas y patriarcales se traducen por una subordinación del placer femenino al placer masculino, o incluso su neutralización al controlar los cuerpos y el goce de las mujeres.
Este artículo se basa en una investigación etnográfica en curso, tanto en zonas rurales como urbanas y periurbanas de Cochabamba. Pondrá énfasis en las relaciones de género y generacionales (más precisamente focalizadas en la familia nuclear) en cuanto al acceso al placer sexual, desvelando la intensa correlación entre dominación, violencias domésticas y sexualidad. Dentro de este contexto, en la última parte, se darán algunas pistas para reflexionar sobre la proacción de las mujeres para buscar y manifestar sus placeres.

\section{EL COMPORTAMIENTO HEDÓNICO DEL HUMANO: CONSIDERACIONES INICIALES ACERCA DE LA NOCIÓN DE PLACER}

El placer es una percepción sensorial del mundo (o su anticipación), inmersa en un estado emocional físico o intelectual agradable y que se inscribe dentro de un marco culturalmente modulado (EASA, taller de estudio alrededor del placer, 2004). También se despliega dentro de un marco social como las relaciones sensoriales en general (Howes, 2003) y de un espacio temporal claramente delimitado. Existen normas y etiquetas que respetar en el mundo sensorial. Por lo tanto, no se puede sentir placer en cualquier lugar, ni como sea, ni con cualquiera. De cierta manera, el placer se controla. Como sucede con otras emociones, los placeres se esconden, son tabúes, son controlados o al contrario exhibidos, cantados, pintados o exaltados. Algunas sociedades consideran el placer como un fin último mientras que otras lo restringen a algunas ocasiones (por ejemplo, la Europa medieval cristiana condenó los placeres del tacto, la dimensión somatosensorial, en beneficio de placeres más intelectuales (Wunsch \& Brenot, 2004: 6).

Investigadores como Spronk (2014), Bozon (1999) o Csordas (1990), y me inscribo dentro de esta perspectiva, sostienen que los actos, las relaciones y las significaciones sexuales son el fruto de una construcción y de transformaciones sociales. Juegan el papel de operadores de categorías sociales e identitarias. Sin embargo, Spronk (2014) añade que no se puede reducir la experiencia erótica a una sola relación simbólica económica, religiosa o política; es necesario introducir el concepto de conocimientos sensoriales encarnados para comprender las relaciones entre los significados sociales de la sexualidad y las sensaciones eróticas. Esta perspectiva demuestra que la significación social del sexo pasa por el cuerpo en su materialidad física, creando de esta manera un sentido particular del ser en relación con el mundo.

En el campo de los estudios andinos, los trabajos sobre el placer son prácticamente inexistentes, con la excepción de Suárez Saavedra (2013a; 2013b), de Sigl (2012) y de Carrasco Gutiérrez \& Gavilán Vega (2014). Los primeros tratan de la sexualidad y particularmente de su historia en Bolivia. Aportan mucha información interesante sobre el placer sexual en este país, desde los tiempos precolombinos hasta el día de hoy. Lamentablemente, se presentan los datos de manera desordenada y sin cronología. Sin embargo, se abordan temas interesantes como el incesto, la violación, la prostitución, la homosexualidad femenina y masculina, los engaños... 
Los otros dos trabajos abordan el erotismo, el placer y la sexualidad en los Andes chilenos y bolivianos. La investigación de Sigl analiza la importancia del humor en la definición del erotismo y de la sexualidad con relación a la fertilidad tanto agrícola como humana (Sigl, 2012: 60). Se basa en bailes donde intervienen personajes con sexualidad desbordante, ambigua, a veces travestis. En cuanto a Carrasco Gutiérrez \& Gavilán Vega (2014), se ubican en una perspectiva particularmente interesante puesto que estudian íntimamente la sexualidad de mujeres de tres generaciones, buscando entender las continuidades en las representaciones, los cambios y las diferencias de género ${ }^{1}$ con relación a la sexualidad.

Fuera de estos estudios, otras pistas pueden contribuir a analizar el placer entendido como parte de la sexualidad. No siempre es fácil distinguir la sexualidad del placer. Los dos acaban entremezclándose: la sexualidad permite la realización del placer y el placer tiene lugar dentro de la sexualidad. En este contexto se encuentra información que proviene de los informes de salud sexual y reproductiva (Castro Mantilla, 2008). Pero los trabajos más numerosos y que aportan mejor luz acerca de las concepciones sobre la sexualidad para explicar el orden social, cósmico y religioso son probablemente los que tratan de la religiosidad mediante metáforas (Harris, 1980; Platt, 1980; 2003; Grebe, 1981; Montes, 1986; Martínez, 1996 ; Albó, 1992; Van Kessel, 1993; Bastien, 1996), y más particularmente para el contexto aymara (Carrasco Gutiérrez, 2010; Carrasco Gutiérrez \& Gavilán Vega, 2006, 2009; Gavilán Vega, 2005).

En un trabajo anterior (Geffroy, 2013a), subrayé que los habitantes de los Andes bolivianos piensan que la Pachamama, la Tierra-Madre, está «excitada», es decir estimula a los vivos y que existe una fuerte correlación entre la fecundidad de la tierra y la sexualidad humana. También se muestra que los muertos quieren gozar de los placeres de la «vida». Esta relación entre sexualidad humana y no humana fue también puesta en evidencia por los estudios de Absi (2005).

Entre estos últimos trabajos, se encuentra un hilo conductor que abarca las relaciones de reciprocidad y de complementariedad cuando se evocan situaciones próximas a lo que llamaríamos placer en español (en quechua, no existe palabra equivalente). En esta perspectiva, se plantea que existe una estrecha relación entre la noción de placer — no necesariamente sexual- y la idea de armonía entre los humanos, el medioambiente, la naturaleza antropomorfizada. El placer viste entonces una dimensión relacional. Esta hipótesis encuentra también eco, por una parte, en las confidencias de las mujeres entrevistadas por Carrasco Gutiérrez \& Gavilán Vega (2014), según quienes la actividad sexual busca, antes que todo, la consolidación de la familia al incitar al hombre a quedarse con su esposa e hijos (obviamente, aquí lo relacional se entiende en una perspectiva hegemónica) por otra parte, se manifiesta en la sexualidad, a menudo encarnada dentro de un sistema de creencias en la cosmovisión andina (Carrasco Gutiérrez \& Gavilán Vega, 2014: 474).
El concepto más amplio del Buen Vivir (que abarca por ende al placer), adoptado en la Constitución boliviana ${ }^{2}$ como uno de los fundamentos de la sociedad, puede ayudar a comprender esta idea. Contiene las nociones de armonía, de equilibrio y de complementariedad. Sin embargo, cabe subrayar que, si bien los roles son complementarios, no significa que sean iguales. Este desequilibrio entre complementariedad e igualdad justamente permite postergar y legitimar cierta forma de dominación de género. Después de esta breve revisión de los estudios sobre el placer y la sexualidad, se indagará sobre la diferencia de acceso a diferentes formas de placer, en función del género.

\section{EL DEBER DE PLACER: GÉNERO, VIOLENCIA, POCIONES} MÁGICAS Y HUMOR

Dentro del fenómeno globalizado de hipersexualización de la sociedad que casi obliga al placer, Bolivia encontró cómo expresarse, en su propia vía, a menudo menos ostentatoria que en otras partes del mundo y que depende mucho del lugar de origen o de la posición en el ciclo vital del(de la) gozador(a). Sin embargo, esta tendencia no es tan fluida y encuentra diferentes obstáculos que exploraremos a continuación.

Cada vez más, se difunden objetos, píldoras, alimentos, imágenes que estimulan lo erótico. En zonas citadinas, por ejemplo, se elogian los beneficios de la pastilla T36 que promete una erección de $36 \mathrm{~h}$, propicia a los excesos carnavalescos; el acceso a la pornografía (venta de CD en la calle) y el consumo de servicios sexuales - también en Internet- se democratizaron, aunque no se puede negar que el énfasis está puesto más bien en el placer masculino.

De manera menos visible, en las comunidades campesinas quechuas, los ancianos se quejan que los jóvenes adopten abiertamente nuevas prácticas amorosas, antes escondidas según ellos, como besarse o agarrarse de las manos públicamente. Existe una influencia de prácticas traídas del extranjero, de las películas y de la intervención de algunas ONG, a su vez generalmente financiadas por organismos externos y multilaterales, en materia de derechos sexuales y reproductivos que promocionan la igualdad entre hombres y mujeres en la sexualidad3. Además, en las últimas dos décadas se ha producido en Bolivia un fuerte proceso migratorio del campo a la ciudad que ha transformado significativamente los modos de vida y, por supuesto, las actitudes y prácticas amorosas y sexuales.

A pesar de estos numerosos cambios, persisten importantes tensiones entre — justamente- estas nuevas prácticas, los proyectos progresistas del gobierno

2 Muy criticado por intelectuales decepcionados por su concretización (Silvia Rivera, 2015: http:// www.erbol.com.bo/noticia/politica/29092015/silvia_rivera_vivir_bien_palabra_hueca_que_no_se_ cumple_para_nada; Jean-Pierre Lavaud 2015: https://blogs.mediapart.fr/jean-pierre-lavaud)

Aunque lo contrario sea también válido: algunas agencias multilaterales ponen trabas a la libre decisión sobre la sexualidad y particularmente sobre el aborto, como USAID. Ver en este número el artículo de Rozée et al. 
actual (por ejemplo, la Ley Integral para garantizar a las mujeres una vida libre de violencia, $2013^{4}$ y la Ley de Identidad de Género, 20165), y la moral impuesta por las Iglesias católica y protestante.

\section{1. Violencia, dominación y sexualidad: ¿un placer diferenciado por género?}

El medio social y cultural influencia la búsqueda del placer. Esta búsqueda puede tener una forma «cooperativa» (no dejar nadie al borde del placer) o puede contenerlo (o incluso prohibirlo), culpabilizando tanto a la gozadora como al gozador. La sociedad andina parece haberse decidido por esta segunda vía y se privilegia una posición «egoísta» (por ejemplo, solo importa el placer masculino). Sin embargo, es necesario matizar esta posición. Es cierto que bajo la presión de los nuevos modos de vida urbanos, los jóvenes cambian de actitud y las mujeres afirman cada vez más su derecho al placer. Parece, como se mostrará más adelante, que las mujeres ancianas de origen rural pueden reivindicar cierto acceso al placer, cuando la menopausia — librándolas de la maternidad y de las obligaciones que la acompañan - les otorga ciertas libertades antes negadas (como ir solas a la chichería, reír abiertamente sobre el sexo y, según algunos testimonios, hasta buscar el goce en la relación sexual). Es como si experimentaran una suerte de masculinización.

Numerosas mujeres que todavía no alcanzan estas libertades viven una sexualidad donde el placer es tabú en el sentido que no se habla de él y que reviste una dimensión moral, generalmente negativa. Se controlan los cuerpos. Esta idea no es nueva. Foucault (1976) ya había mostrado la relación entre la sexualidad y la vida moral y ética de los individuos. La sociedad occidental, decía, organizó un nuevo tipo de poder, el biopoder, que se ejerce sobre la vida y los cuerpos de la población. Lo que es particularmente evidente, en la sociedad boliviana, en las manifestaciones de pudibundez traídas por la Contrarreforma española que marcó fuertemente las prohibiciones sexuales.

La búsqueda del placer puede llevar a situaciones azarosas y hasta de violencia ya sea física o más sutil y tal vez más nociva todavía bajo la forma de relaciones de fuerzas psicológicas, desde el machismo, el patriarcado, las humillaciones pero también las violencias conyugales —-sexuales o no-, las violaciones, os incestos, la imposición del placer masculino en detrimento del goce de la mujer... Obviamente, estas formas de dominación no garantizan el acceso a un placer equivalente entre los géneros. Diamond (2000) defiende que el placer es la motivación primera del acto sexual, pero uno se puede preguntar si la felicidad sexual femenina representa una meta en la sociedad andina (puesto que la búsqueda del placer femenino es una noción fuertemente cultural que no

4 http://www.migracion.gob.bo/upload//348.pd

https://sistemas.mre.gov.br/kitweb/datafiles/SantaCruz/pt-br/file/bolivia\%20-\%20ley\%20807\%20 -\%20ley\%20de\%20identidad\%20de\%20género\%20-\%2022\%20mai\%2016.pd siempre existió). Por cierto, según las confidencias que se recogieron de mujeres campesinas o citadinas, corroboradas por los estudios en el Alto de Camacho et al (1997: 32), no todas gozan físicamente durante la relación sexual y probablemente no sea su meta. Ellas sienten que el coito es no solamente una obligación y una estrategia para tener paz en su matrimonio, pero también una forma de violencia o de depredación, pues tienen la sensación que les quitaron una parte de sí.

Se escribió mucho sobre la violencia de género en los Andes (por ejemplo, Harvey \& Gow, 1994). Aquí, entenderemos por violencia contra las mujeres todo acto sexista que tiene o que podría tener por resultado un daño o un sufrimiento físico, sexual, psicológico, emocional, sea que ocurra en el campo público o privado. Notemos que no existe en quechua un vocablo que corresponda precisamente a la noción de violencia tal como se emplea en la sociedad occidental moderna. En cambio, se encuentra en el primer diccionario quechua de Holguín (2007 [1608]), la entrada «Violar doncella» que se traduce por Huarmicta ppaquini hutcuni chancani, ccacuni y también «Violadada», Chancascca ppaquiscca hutcuscca. Lo que muestra que la práctica tenía suficiente importancia como para ser reportada en un diccionario. Esta definición subraya también que no se castigaban moralmente las violaciones de mujeres que ya no eran doncellas - por definición puras y vírgenes-. Esto sigue de actualidad en vista de la recurrencia de las violaciones, particularmente las incestuosas, que quedan impunes.

Parece prudente dividir los actos violentos en la sexualidad en dos categorías de diferentes grados. La primera agrupa los casos extremadamente violentos, tales como los feminicidios o las violaciones y la segunda, prácticas y gestos agresivos o de una violencia menos visible 6 . Veamos algunos espacios donde se ejerce esta violencia/agresividad por orden de intensidad.

Previamente, cabe destacar que Bolivia ocupa el segundo lugar en América Latina en términos de número de casos y de denuncias de violencia sexual hacia las mujeres, niñas y adolescentes ${ }^{7}$ la primera en lo que concierne a la violencia hacia la mujer8. A esta constatación, cabe añadir que, tanto en zona urbana como en situación rural, se recogieron varios testimonios sobre experiencias de violaciones incestuosas, también documentadas por Suárez Saavedra (2013a; 2013b). Durante la investigación llevada a cabo en El Alto, en 1996, por Camacho et al. (1997: 48-49), un 85\% de las mujeres entrevistadas declararon haber sufrido un abuso sexual o verbal y de ellas, la tercera parte fue forzada por su pareja a tener relaciones sexuales mediante el uso de la violencia (Camacho et al., 1997: 36).

Lamentablemente, las violencias no son únicamente de índole sexual. Penelope Harvey \& Peter Gow (1994) describen las múltiples formas de violencia sufridas por las mujeres de la ciudad de E Alto, como las humillaciones, las privaciones de recursos (dinero o movilidad), entre otras.

Ver por ejemplo :

http://internacional.elpais.com/internacional/2014/08/24/actualidad/1408917187_424590.html

Ver por ejemplo:

http://internacional.elpais.com/internacional/2014/08/24/actualidad/1408917187_424590.htm 
Fuera de la esféra doméstica, las violaciones son muy frecuentes en numerosas instituciones públicas 9

Una forma de violencia de menor intensidad es particularmente notable en la reproducción de la dominación masculina especialmente mediante el machismo, el patriarcado y las diversas instituciones que la reproducen, omnipresentes en todas las esferas de la sociedad, incluyendo las Iglesias y el Estado. En iSomos machistas los cochabambinos? Ewel Rengel (1998) analiza las influencias de los estereotipos de género en la sociedad boliviana y en particular las contradicciones inherentes al machismo. En efecto, se espera que los hombres velen por el bienestar de sus familias y a la misma vez que afirmen su masculinidad, a menudo mediante conductas sexuales irresponsables y hasta violentas, mientras que enmascaran sus vulnerabilidades emocionales y físicas.

Uno de los factores que legitiman estas relaciones de dominación es la violencia hacia las mujeres por parte de los dirigentes políticos, lo que tiene un efecto devastador. Desde su llegada al poder, el presidente boliviano, Evo Morales, recurre sin cesar a bromas groseras, denigrantes, machistas y de carácter sexual hacia las mujeres. Por ejemplo, preguntó varias veces a mujeres que manejan máquinas perforadoras en campos petroleros si se «dejaban perforar»; en otras oportunidades se jactó de embarazar a todas las mujeres de los pueblos que visita; otra vez, aconsejó a los chicos que hayan embarazado a chicas muy jóvenes refugiarse en el servicio militar donde encontrarían cobijo. El hecho de que uno de los hombres más influyentes del país pueda permitirse tales comentarios, infringiendo las leyes y la Constitución, es significativo de una cierta forma de tolerancia. Esta benevolencia, a veces, hace sonreír a la clase política sin provocar réplicas importantes y organizadas, salvo desde algunos sectores de la sociedad, como intelectuales y feministas.

Las relaciones domésticas son también a menudo el teatro de violencia. Forma parte de la experiencia sexual de las mujeres andinas (Carrasco Gutiérrez \& Gavilán Vega, 2014: 471). Se arraiga en las relaciones de género y de jerarquía que las mujeres incorporaron desde su más tierna edad cuando admiten que se tiene que respetar a los mayores así como a los hombres cuyo deber, a su vez, es protegerlas. La estabilidad de su relación conyugal depende de esta jerarquía. En este contexto, los hermanos mayores tienen derecho de pegar a sus hermanitas (Harvey \& Gow, 1994: 69).

Es muy común que las mujeres jóvenes experimenten una violación durante sus primeras relaciones sexuales (Carrasco Gutiérrez \& Gavilán Vega, 2014). Después de su matrimonio, la violencia sexual sigue, particularmente cuando el hombre se encuentra borracho, cuando vuelve a casa después de una ausencia prolongada y también durante el embarazo (Carrasco Gutiérrez \& Gavilán Vega, 2014: 471). Los maridos controlan a las mujeres; las acusan de engañarlos o de buscar amantes cuando no desean relaciones sexuales, cuando llevan nueva ropa, cuando ríen

9 Ver por ejemplo el caso de la mujer que fue violada por diputados en 2013: http://www. huffingtonpost.es/2013/01/18/las-camaras-graban-como-u_n_2504764.html con otros hombres, cuando no respetan cierta proxemia, cuando miran a los ojos, cuando se lavan el cabello (Carrasco Gutiérrez \& Gavilán Vega, 2014: 472). Entonces se manifiestan los celos, considerados por las mujeres como uno de los mayores problemas de pareja que se exteriorizan, a menudo, a través de formas violentas.

Otra forma de violencia sufrida por las mujeres entrevistadas concierne a las relaciones sexuales en estado de ebriedad y que pueden tener desenlaces patéticos: «Hay mujeres que no saben a quién acusar de ser el padre del bebé», me decía una habitante de Qhoari. En efecto, en esta región, es común que las jóvenes beban durante las fiestas comunitarias y luego se embaracen contra su voluntad (puesto que a veces ni recuerdan quién es el autor de los hechos). Durante un trabajo de investigación previo (Geffroy, 2013a), percibí -aunque no desarrollé el tema - que el hombre borracho actúa de manera violenta no por la magia de una mano invisible, sino más bien porque busca la ebriedad para realizar estos actos más fácilmente. Creo que existe una intencionalidad en el gesto del beber y que, aunque no lo formulen verbalmente, los hombres ebrios tienen alguna consciencia de lo que hacen. Lo que se sugiere es que el culpable de una violación puede haber bebido justamente para poder violar. Además, en la frase mencionada por esta interlocutora, se percibe una noción de confrontación al querer acusar a un hombre de un embarazo.

Tanto Harvey \& Gow (1994) como Paulson \& Bailey (2003) se sorprenden que, aun conociendo sus derechos, las mujeres acepten las situaciones violentas por costumbre, por miedo, e incluso consideren que la violencia que padecen es normal. No piensan que sus maridos sean directamente responsables, incluso en casos extremos (Harvey \& Gow, 1994: 67). Entonces, es necesario interrogarse sobre las razones que impiden a las mujeres cuestionar su situación. ¿Cuál es el grado de violencia que están preparadas a aceptar por parte de sus esposos? Tanto Harvey \& Gow como Carrasco Gutiérrez \& Gavilán Vega (2014: 472) afirman que esta confrontación es percibida por ellas como una consecuencia desagradable del matrimonio pero necesaria para favorecer la estabilidad de la familia y que el sexo, aun en condiciones violentas, es parte de las obligaciones matrimoniales.

Asimismo, la búsqueda del placer privilegia al del hombre en detrimento, o incluso en la negación o hasta prohibición de aquel de la mujer. Se debe a que el derecho al placer sexual es una construcción social y, por lo tanto, es rígidamente controlado (cf. lo antes mencionado sobre el biopoder según Foucault); este hecho tiene una fuerte incidencia sobre el comportamiento sexual. En la región andina boliviana, hombres y mujeres no siempre aceptan la expresión del goce de la mujer. A menudo, el hombre desea guardar el control sobre el cuerpo de la mujer (no solamente en el placer sino también en todo el campo de la sexualidad). Por ejemplo, las campañas de esterilización son a veces conflictivas, así como la planificación familiar, puesto que numerosos hombres imaginan que sus mujeres aprovecharán lo aprendido para engañarles luego (cf. Rozée et al., en este mismo volumen). Otros testimonios masculinos (Camacho et al., 1997) insisten sobre su deseo de que sus mujeres se comporten «como monjas» durante el acto sexual, sin ninguna iniciativa, bajo pena de sentirse humillados y celosos. 
Cabe resaltar que, según el estudio de Camacho et al. (1997), en la ciudad de El Alto, la mayoría de las mujeres afirman no sentir placer en la relación, citando la vergüenza, el miedo de los embarazos no deseados y la violencia como factores limitantes (Camacho et al., 1997: 32). Las dos terceras partes de las mujeres interrogadas dejan a sus esposos tomar las iniciativas sexuales. Por el contrario, los hombres confiesan un sentido más importante del control y de la satisfacción en sus experiencias sexuales que las mujeres: expresan más fácilmente lo que les gusta o no en la relación sexual (Camacho et al., 1997: 33). Estos desfases en el acceso al placer alientan a los hombres a buscar la satisfacción sexual en relaciones extramaritales, ganando de paso cierto status ante sus pares (Carrasco Gutiérrez \& Gavilán Vega, 2014: 489). No hay que confundir la sexualidad matrimonia reproductiva con la sexualidad recreativa.

En la ciudad de Cochabamba, las mujeres de la clase media indicaron en el mismo estudio que las buenas esposas no deberían expresar su deseo sexual ni mostrar sus habilidades (Camacho et al., 1997: 34-35). Según una encuesta realizada entre las aymaras del norte de Chile (Carrasco Gutiérrez \& Gavilán Vega, 2014), como se ha mencionado anteriormente, las mujeres aceptan las relaciones sexuales para retener a sus maridos — quienes, en el caso contrario, podrían buscar amantesy preservar de esta manera el equilibrio familiar, tan importante en la sociedad andina donde el divorcio sigue visto como un fracaso y es fuente de oprobio, mientras se percibe a las mujeres solteras como desprotegidas. Entonces, para ellas, una vida sexual activa equivale a alentar a su esposo a quedarse al lado de su familia. Por lo tanto, hay que agradar al hombre, hacer que se sienta feliz. Durante las relaciones sexuales, las mujeres juegan más fácilmente un papel pasivo, de receptoras, en las reglas de una actitud sumisa aprendida en la niñez, socialmente y culturalmente establecidas (Carrasco Gutiérrez \& Gavilán Vega, 2014: 471). Si bien algunas reconocen que pueden sentir placer, siempre enfatizan la primacía de la familia y de su reproducción al tener una relación sexual.

Me pregunto entonces si las constricciones morales y psicológicas, particularmente fuera de la relación matrimonial estable, especialmente en el caso de jóvenes que todavía no son concubinos o entre amantes, no representarían un freno para aceptar la sensación física del placer. Asimismo, en el momento del coito, el miedo al embarazo probablemente no sea propicio para favorecer un placer sexual intenso, así como los encuentros para copular a escondidas, en los matorrales y en el frío.

En suma, dentro del contexto cultural andino, la sexualidad femenina está canalizada hacia la conyugalidad y la procreación; la búsqueda del placer femenino per se es reprimida.

La construcción social de la sexualidad masculina como depredación se expresa mediante cierta agresividad en los rituales de seducción. Existe en el rapto de mujeres, el suwanaku, y en particular cuando ocurre por la fuerza -lo que no siempre es el caso (Geffroy, 2013a)—, cuando los enamorados se insultan o se tiran piedras (Harvey \& Gow, 1994), durante los bailes rituales donde los jóvenes se desafían mediante coplas o físicamente hasta hacerse caer: una manera de acercar los sexos y favorecer tanto la fecundidad humana como la fertilidad de la naturaleza antropomorfizada (Van den Berg, 1989: 63; Sigl, 2012: 57).

Finalmente, la dominación de género puede manifestarse en la utilización de la sexualidad como forma de castigo. No es raro descubrir en las comunidades andinas la existencia de humillaciones para aquellos quienes, por ejemplo, pegan a sus mujeres estando borrachos, aquellos que faltan el respeto a sus padres o a los representantes políticos. Se les obliga a disfrazarse de mujer (Harvey \& Gow, 1994: 68). Aquello parece demostrar que la sexualidad elevada como arma de punición comporta en sí los gérmenes de la agresividad y de cierta forma de violencia además de un menosprecio intrínseco hacia el género femenino.

Estas actitudes se interiorizan desde el nacimiento y luego se transmiten por la familia, por la escuela, las Iglesias y por los medios de comunicación. Vimos que, aun cuando las mujeres conocen sus derechos, por costumbre o por miedo, consideran que la violencia que sufren es normal.

A pesar de estas limitantes, como numerosos estudios de género lo subrayaron, las mujeres no son forzosamente meras actrices pasivas de imposiciones masculinas (Querrien \& Selim, 2015; Absi, 2011 quien subraya que las prostitutas pueden utilizar la sexualidad mercantil para subvertir la dominación masculina). Imaginan vías que les permitan conocer cierta forma de realización personal en la relación sexual y lo que abarca. Placer que consiste, en primer lugar en asegurar la reproducción familiar y que se concretiza, entre otros aspectos, mediante el humo y ciertas transgresiones como tener un amante, o aprovechar el placer después de la menopausia o volverse viudas, cuando los hijos ya se fueron de casa.

\section{OTRAS VÍAS DE PLACER: LAS RESPUESTAS FEMENINAS}

Entonces, si las mujeres pretenden no sentir placer en el momento de la relación sexual, ¿cómo explicar esta abundancia de objetos (fabricados tanto por mujeres como por hombres) que presentan atributos sexuales exagerados, de recetas (el «cardan caldito» de testículos de toro - también empleado contra la resaca- o también el consumo de mariscos), de polvos, de amuletos, de perfumes, de algunas plantas como la maca (Lepidium meyeni) conocida como el «Viagra andino», la papalisa, este pequeño tubérculo color fucsia y amarillo, de piedras, de lanas de colores, de perfumes con nombres intrigantes tales como Sígame, sígame o también de velas para exaltar la libido? (fig. 1). Estos artefactos se encuentran en La Paz, en la calle Sagarnaga (también conocida como «calle de las brujas») como en numerosos otros mercados del país, donde se venden en corredores enteros con olores y colores tan particulares. 


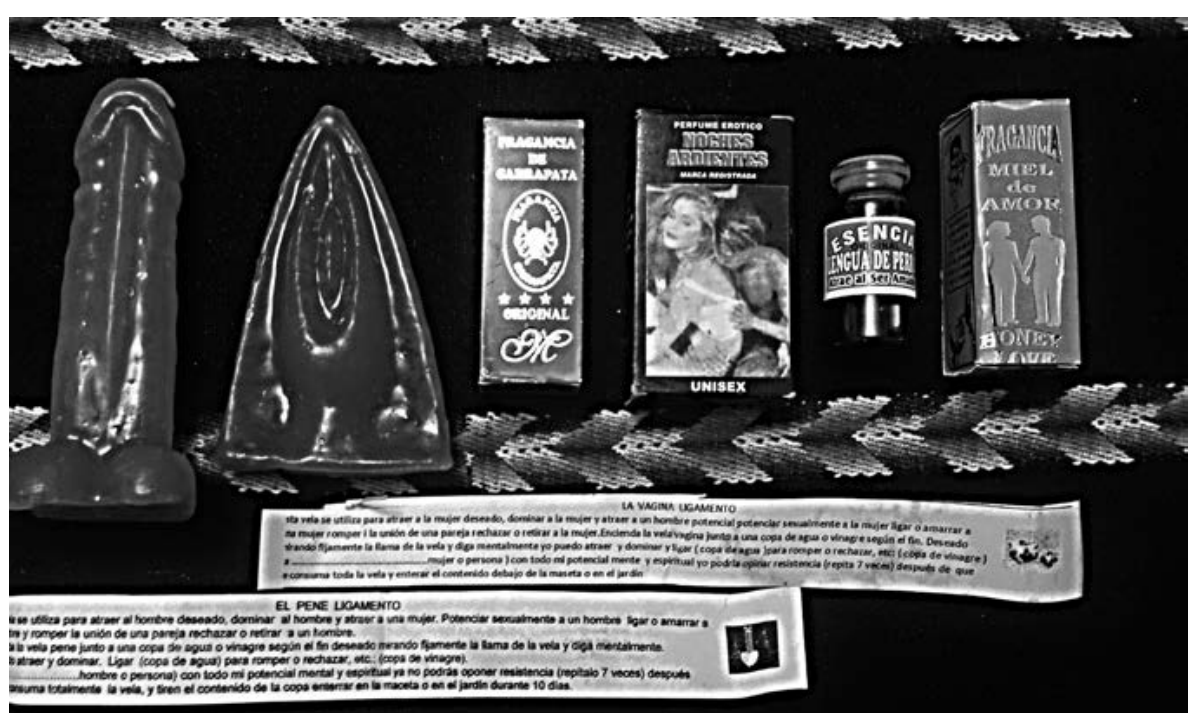

Figura 1 - Perfumes, pociones y velas (con los rezos a recitar) utilizados en la magia para el

(C) Céline Geffroy, 2016

Pueden haber varias explicaciones para elucidar esta cuestión. Tal vez, justamente buscan en estos amuletos y recetas el poder de encontrar el placer que, potencialmente, sienten que podría volverse alcanzable. Tal vez sea para atrapar al hombre al darle placer, lo que, se vio, es una de las metas de las relaciones sexuales... Asimismo, cabe destacar que el placer sexual parece variar en función del ciclo vital. Así, las mujeres más ancianas entrevistadas por Carrasco Gutiérrez y Gavilán Vega reconocieron que la sexualidad es el fruto de un aprendizaje común con el marido, algunas admitiendo incluso que buscan la satisfacción en la relación sexual (Carrasco Gutiérrez \& Gavilán Vega, 2014: 471).

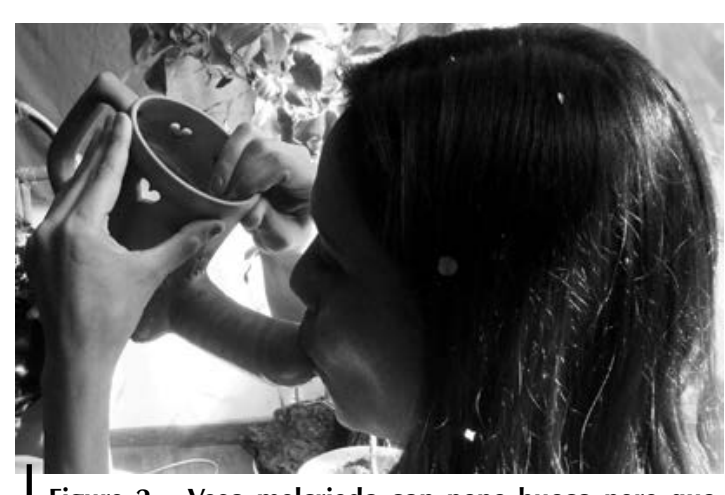

Figura 2 - Vaso malcriado con pene hueco para que las mujeres puedan beber directamente del sexo

๑) Céline Geffroy, 2014
Asimismo, se cree percibir en los testimonios cierta forma de regocijo ligado al humor -lo que Sigl (2012) también describió precisamente- alrededor de la sexualidad. Encontramos objetos con representaciones sexuales que hacen reír sobre todo a las mayores, como los vasos malcriados que representan las partes genitales tanto de hombres (fig. 2) como de mujeres (fig. 3). También ríen de las bromas con carácter sexual que hacen algunos bailarines en el carnaval o en las festividades de Todos Santos. Las burlas públicas sobre las pobres prestaciones sexuales o sobre el tamaño ridículo del pene son plétoras en las coplas

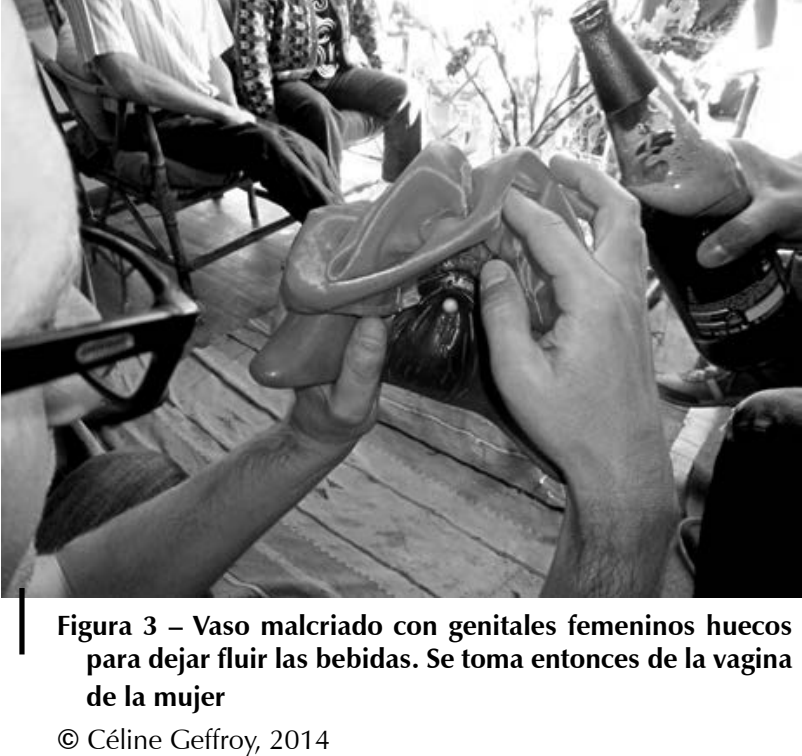

tanto de Carnaval como durante algunos rituales de Todos Santos (Geffroy, 2013b) y solo tienen competencia en las bromas de connotaciones sexuales que suelen hacer las mujeres de edad avanzada cuando se encuentran entre sí. Estas burlas, abiertas, sin piedad, parecen un exutorio a las tensiones acumuladas. El humor alrededor del placer manifiesto o escondido supone desplazar el placer físico per se al ámbito del discurso entre personas no implicadas en el coito (entre mujeres) y postergarlo en el tiempo. Esta actitud permite reírse de la sexualidad aun cuando puede tornarse en un factor de violencia.

Aunque no siempre admiten un placer físico compartido en su unión matrimonial, algunas mujeres aseguraron mantener relaciones satisfactorias con amantes. Se puede suponer que se sienten más a sus anchas fuera de la ebriedad para sentir placer y que con la transgresión aparece también el placer. Podemos ir más allá de esta suposición conjeturando que, una vez consolidada la familia, las mujeres como los hombres buscan fuentes de placer en relaciones extraconyugales.

\section{CONCLUSIÓN}

El placer sexual en Bolivia no se experimenta al margen de un mundo globalizado cada vez más erotizado. Sin embargo, los tabúes, las normas, las prohibiciones (y lo permitido) están intensamente marcados por el poder del machismo y del patriarcado. En este artículo, se buscó analizar estas relaciones de autoridad de manera más precisa dentro de la institución familiar.

En este contexto, se intentó proponer algunas pistas para analizar la diferencia de acceso al placer en función del género. La situación de las mujeres no es nada 
envidiable. Sufren, a menudo, las consecuencias de un placer masculino egoísta y descontrolado y son víctimas de relaciones sobre su cuerpo y sus emociones.

A pesar de estos múltiples obstáculos, a su manera, las mujeres logran afirmar sus deseos y encontrar cómo experimentar ciertas formas de placer. Otorgar mayor permisividad y aceptar la igualdad ante el placer, aunque cada uno a su manera, favorecerá un bienestar compartido y, por lo tanto, probablemente multiplicado. En efecto, la puesta en subordinación y/o al borde del placer de la mitad de la población puede volverse nefasta cuando se sabe que nuestro sistema hedónico juega un papel en la regulación de los comportamientos y que las últimas investigaciones, tanto en ciencias sociales como en ciencias de la vida, resaltan la importancia de la felicidad y del placer sobre la salud mental y física de los individuos.

\section{Referencias citadas}

ABSI, P., 2005 - Los ministros del diablo: el trabajo y sus representaciones en las minas de Potosí, 339 pp.; La Paz: PIEB, IFEA, IRD, Embajada de Francia en La Paz.

ABSI, P., 2011 - De la transgresión a la subversión. El valor del dinero en los prostíbulos de Bolivia. In: Capitalismo y Pornología. La producción de los cuerpos sexuados (I. Pavez \& L. Kraushaar, eds.): 379-403; San Pedro de Atacama: Quilqa, Universidad Católica del Norte.

ALBÓ, X., 1992 - La experiencia religiosa aymara. In: Rostros Indios de Dios (M. Marzal, coord.): 81-130; La Paz: Hisbol.

BALANDIER, G., 1984 - Le sexuel et le social. Lecture anthropologique. Cahiers internationaux de sociologie, vol. 76: 5-19; París: Les Presses universitaires de France.

BASTIEN, J. W., 1996 - La montaña del Cóndor. Metáfora y Ritual en un Ayllu Andino, 253 pp.; La Paz: Hisbol.

BOZON, M., 1999 - Les significations sociales des actes sexuels. Actes de la recherche en sciences sociales, Vol. 128: 3-23.

CAMACHO, A., RUEDA, J., ORDOÑEZ, E. \& LÓPEZ, R., 1997 - Las mujeres de El Alto se descubren a sí mismas: impacto de la regulación de la fecundidad sobre la estabilidad de la pareja, la sexualidad y la calidad de vida; La Paz: Family Health International: Bolivia Field Office.

CANDAU, J., 2017 - The Theuth effect. What does culture do to our brains? UNESCO Encyclopedia of Life Support Systems (EOLSS) (en prensa).

CARRASCO GUTIÉRREZ, A. M., 2010 - Paradigmas éticos y morales en la construcción de la sexualidad de hombres y mujeres indígenas y no indígenas en el norte de Chile. Revista Nuevas Tendencias en Antropología, 1: 108-126.

CARRASCO GUTIÉRREZ, A. M. \& GAVILÁN VEGA, V. T., 2006 - Sexualidad y género: La unidad de lo femenino y lo masculino como símbolos de reproducción y fertilidad entre los Aymará del norte de Chile In: Imaginarios, Identidades e Historias. Miradas desde la Antropología del Género (L. Rebolledo, P. Tomic \& E. Garduño, comps.): 169-186; México: Universidad Autónoma de Baja California.
CARRASCO GUTIÉRREZ, A. M. \& GAVILÁN VEGA, V. T., 2009 - Representaciones del cuerpo, sexo y género entre los aymara del norte de Chile. Chungara. Revista de Antropología Chilena, 41: 83-100.

CARRASCO GUTIÉRREZ, A. M \& GAVILÁN VECA V. T, 2014 - Significados y prácticas de la sexualidad en tres generaciones de mujeres aymaras del norte de Chile. Interciencia, Vol. 39 n. ${ }^{\circ}$ 7: 468-475.

CASTRO MANTILLA, M. D., 2008 - Los determinantes socioculturales en la salud sexual y reproductiva de mujeres indígenas. Revista Umbrales en Ciencias Sociales, $\mathbf{n} .{ }^{\circ} \mathbf{1 8}$ 203-235.

CSORDAS, T., 1990 - Embodiment as a paradigm for anthropology. Ethos, 18: 5-47.

DIAMOND, J., 2000 - Le troisième chimpanzé. Essai sur l'évolution et l'avenir de l'animal humain, 466 pp.; París: Gallimard.

EWEL RENGEL, R., 1998 - iSomos machistas los cochabambinos?, 177 pp.; Cochabamba: Odec. FOUCAULT, M., 1976 - Histoire de la sexualité. Tome 1: La volonté de savoir; París: Gallimard. GAVILÁN VEGA, V. T., 2005 - Representaciones del cuerpo e identidad étnica en la población indígena del norte de Chile. Estudios Atacameños, 30: 135-148.

GEFFROY, C., 2013a - Boire avec les morts et la Pachamama. Une anthropologie de I'ivresse rituelle et festive dans les Andes boliviennes; Niza: Universidad de Nice Sophia-Antipolis. Laboratorio de Antropología y de Psicología Cognitivas (LAPCOS). Tesis doctoral inédita escrita bajo la dirección del Profesor Joël Candau.

GEFFROY, C., 2013b - La sensualidad de la wallunk'a. Diálogo con los muertos y rituales de fertilidad. Arqueoantropológicas, n. ${ }^{\circ}$ 2: 79-96; Cochabamba, Bolivia: Revista del Instituto de Investigaciones Antropológicas (INIAM) del Museo ArqueológicoUniversidad Mayor de San Simón (UMSS).

GONZÁLEZ HOLGUíN, D., 2007 [1608] - Vocabvlario de la Lengva General de todo el Perv llamada Lengua Qquichua, o del Inca. Digitalizado por Runasimipi Qespisqa Software (http://www.runasimipi.org)

GREBE, M., 1981 - Cosmovisión aymara. Revista Santiago, 1: 61-79.

HARRIS, O., 1980 - The power of signs: gender, culture and the wild in the Bolivian Andes. In: Nature, Culture and Gende (C. Macormarck \& M. Strathern, eds.): 70-94; Cambridge: Cambridge University Press.

HARVEY, P. \& GOW, P., 1994 - Sex and Violence: Issues in Representation and Experience, 197 pp.; Londres: Routledge.

HOWES, D., 2003 - Sensual Relations: Engaging the Senses in Culture and Social Theory, xxvi + 283 pp.; Ann Erbor: University of Michigan Press.

MARTíNEZ, G., 1996 - Saxra (diablo)/Pachamama, Música, Tejido, Calendario e Identidad entre los Jalq'a. In: Cosmología y Música en los Andes (M. Bauman, ed.): 283-310; Madrid: Iberoamericana-Vervuert.

MONTES, F., 1986 - La Máscara de Piedra. Simbolismo y Personalidad Aymaras en la Historia; La Paz: Quipus.

PAULSON, S. \& BAILEY, P., 2003 - Culturally Constructed Relationships Shape Sexual and Reproductive Health in Bolivia. Culture, Health \& Sexuality, Vol. 5, n. ${ }^{\circ}$ 6: 483-498; Taylor \& Francis, Ltd. Stable URL: http://www.jstor.org/stable/4005380

PLATT, T., 1980 - Espejos y maíz. El concepto de Yanantín entre los Macha de Bolivia. In: Parentesco y Matrimonio en los Andes (E. Mayer \& R. Bolton, eds.): 139-182; Lima: Pontificia Universidad Católica del Perú. 
PLATT, T., 2003 - El feto agresivo. Parto, formación de la persona y mito-historia en los Andes. Estudios Atacameños, 22: 127-155.

QUERRIEN, A. \& SELIM, M., 2015 - La libération des femmes : une plus-value mondiale, 273 pp.; París: L'Harmattan.

SIGL, E., 2012 - Erotismo, sexualidad y humor en las danzas del Altiplano boliviano. «Maguaré», 26 (2): 51-86. Número temático dedicado a Ritos y Juegos. http://www.bdigital.unal.edu.co/36834/\#sthash.ih4wSXFw.dpuf Maguaré

SPRONK, R., 2014 - Sexuality and subjectivity: erotic practices and the question of bodily sensations. Social Anthropology/Anthropologie Sociale, 22 (1): 3-21. (C) 2014 European Association of Social Anthropologists 3, doi:10.1111/1469-8676.12055.

SUÁREZ SAAVEDRA, F., 2013a - El placer de los placeres. Historia de la sexualidad en Bolivia, Tomo I desde la época prehispánica hasta fines de la Colonia, 302 pp.; Sucre: sin editor.

SUÁREZ SAAVEDRA, F., 2013b - El gozo de los gozos. Historia de la sexualidad en Bolivia. Tomo II: desde inicios de la republica hasta nuestros días, 240 pp.; Sucre: sin editor.

TIGER, L., 2003 - À la recherche des plaisirs, 439 pp.; París: Petite Bibliothèque Payot.

VAN DEN BERG, J., 1989 - 'La tierra no da así no más'. Los ritos agrícolas en la religión de los aymara-cristianos, xviii + 291 pp.; Amsterdam: CEDLA.

VAN KESSEL, J., 1993 - Pachamama, La Virgina: La que Creó el Mundo y Fundó el Pueblo, 43 pp.; Puno: CIDSA.

WUNSCH, S. \& BRENOT, P., 2004 - Les fondements neurobiologiques de la sexualité. Neurobiologie du plaisir. Revue Européenne de Sexologie; Sexologies, (XIII), 50 : 4-16.

\section{Página web sobre la noción de placer}

workshop EASA http://www.easaonline.org/downloads/easa04/ws6.pdf 